\title{
Precise recursive formula for calculating spot size in optical waveguides and accurate evaluation of splice loss
}

\author{
Yasuo Kokubun and Shuichi Tamura
}

\begin{abstract}
The spot size of a single-mode waveguide was defined from the viewpoint of a least-squares fit of the field profile to a Gaussian profile. The field profile was expanded in terms of Hermite-Gaussian functions, and a new precise recursive formula for calculating the spot size was derived. It was shown that our formula is equivalent to the best fitting of the offset coupling loss to that of a Gaussian profile and keeps its form against the Fourier transform that corresponds to the diffraction in the same manner as the Gaussian profile. The accuracy of conventional formulas and our new spot-size formula was compared with the exact value defined from the viewpoint of a least-squares fit to a Gaussian profile, and it was shown that our recursive formula is the most accurate of the approximate formulas. Next we proposed a new formula for calculating the splice loss between two waveguides and showed that our formula is more accurate than the conventional one, which involves only the spot size. (1995 Optical Society of America
\end{abstract}

\section{Introduction}

Recently the demand for monolithic integration of various waveguide-type devices has been increased. To achieve a low splice loss between optical devices that have different spot sizes, such as laser diodes, modulators, switches, and single-mode fibers, many types of spot-size transformers have been developed..$^{1-7}$ The spot size is a fundamental parameter for evaluating the spread of an optical field of single-mode waveguides or optical devices and is useful for evaluating the splice loss between two different singlemode waveguides. Accordingly, it is important to evaluate the accurate value of the spot size for the precise design of integrated photonic circuits that involves many waveguide-type devices.

For a Gaussian beam, the spot size is defined as $1 / e^{2}$ half-width of the power distribution or $1 / e$ half-width of the field profile. Because the field profile of the fundamental mode of a single-mode waveguide is different from that of a Gaussian beam, the spot size is evaluated by the approximation of the field profile of the waveguide to the Gaussian profile. Several formulas for evaluating the spot size have

The authors are with the Division of Electrical and Computer Engineering, Faculty of Engineering, Yokohama National University, 156 Tokiwadai, Hokogayaku, Yokohama, 240 Japan.

Received 9 June 1995; revised manuscript received 9 June 1995. 0003-6935/95/306862-12\$06.00/0.

(c) 1995 Optical Society of America. been proposed, such as the formula of Villuendas et $a l .{ }^{8}$ which is easily derived from the coordinate transformation of Petermann's formula ${ }^{9}$ type II and a formula based on the quadratic moment of the power distribution. ${ }^{10,11}$ The $1 / e^{2}$ half-width of the power distribution is sometimes used as a brief evaluation. However, there has been no discussion on the mathematical strictness and the accuracy of these formulas. Although Petermann's formula type II is widely used for calculating the spot size (or mode field diameter) of single-mode fibers, its mathematical appropriateness or uniqueness has not been proved, because this formula was derived from the stationary expression of the Helmholtz wave equation and can be considered as some other stationary expression. ${ }^{12}$ In addition, a Gaussian profile keeps its form against the Fourier transform that corresponds to the diffraction of an optical beam, whereas the above formulas change their forms. Therefore these formulas are not mathematically self-consistent as approximate formulas to the Gaussian profile.

Another viewpoint of the strictness of spot-size formula is the accuracy of the evaluation of splice loss between two different waveguides. An approximate formula for calculating the splice loss between two waveguides with offset can be easily derived from the overlap integral of two Gaussian profiles that have offset. However, even if the spot sizes of two waveguides are equal, it sometimes happens that the two waveguides have different field profiles, like the com- 
bination of conventional waveguides that have different $V$ values and antiresonant reflecting optical waveguides. ${ }^{13}$ In this case, the coupling loss (splice loss) is not 0 , although it is evaluated to be 0 from the spot size. This fact implies that another parameter is required for evaluating the splice loss precisely.

In this paper we propose a precise recursive formula for calculating the spot size in optical waveguides and derive an analytical expression of the splice loss between two waveguides with offset. The accuracy of these formulas is discussed. Because the two-dimensional Gaussian profile can be expressed by the product of one-dimensional Gaussian profiles in the $x$ and the $y$ directions, we concentrate our discussions on the one-dimensional case.

\section{Derivation of Formulas}

A. New Formula for Calculating Spot Size in Optical Waveguides

We define a one-dimensional transverse field profile by real function $f(x)$. The optical power is normalized as follows:

$$
\int_{-\infty}^{\infty}[f(x)]^{2} \mathrm{~d} x=1
$$

When $f(x)$ is expanded in terms of Hermite-Gaussian functions that have a spot size $w, f(x)$ is expressed by

$$
f(x)=\sum_{i=0}^{\infty} C_{i} N_{i} H_{i}\left(\sqrt{2} \frac{x}{w}\right) \exp \left[-\left(\frac{x}{w}\right)^{2}\right],
$$

where $C_{i}$ is the expansion coefficient, $N_{i}$ is the normalization constant, and $H_{i}(x)$ is the Hermite polynomial of the $i$ th order. These are described by

$$
\begin{aligned}
C_{i} & =N_{i} \int_{-\infty}^{\infty} f(x) H_{i}\left(\sqrt{2} \frac{x}{w}\right) \exp \left[-\left(\frac{x}{w}\right)^{2}\right] \mathrm{d} x \\
N_{i} & =\frac{1}{\left(2^{i} i ! w \sqrt{\frac{\pi}{2}}\right)^{1 / 2}}
\end{aligned}
$$

Using Eq. (1) and the orthonormality of the HermiteGaussian function, we obtain

$$
\sum_{i=0}^{\infty}\left(C_{i}\right)^{2}=1
$$

In this section the expansion up to second order is used, and the field profile of a fundamental mode in a waveguide is assumed to be symmetric with respect to the optical axis, i.e., first-order coefficient $C_{1}$ is assumed to be 0 .

Here we define $f_{N}(x)$ as the approximate function of $f(x)$ in terms of the Hermite-Gaussian series expansion up to the $N$ th order. Let us suppose that the mean-squared error between $f_{N}(x)$ and the exact function $f(x)$, which is defined by

$$
I_{\text {Field }}(w)=\int_{-\infty}^{\infty}\left[f(x)-f_{\mathrm{N}}(x)\right]^{2} \mathrm{~d} x .
$$

When the spot size $w$ is constant, the expansion coefficient $C_{i}$ that minimizes Eq. (6) is given by Eq. (3), and it is independent of $N$. This fact means that the series expansion in terms of an orthonormal set of functions gives the least-squares fit for the $N$ th order. Equation (6) can be rewritten as

$$
\begin{aligned}
I_{\text {Field }}(w)= & \int_{-\infty}^{\infty}[f(x)]^{2} \mathrm{~d} x+\int_{-\infty}^{\infty}\left[f_{N}(x)\right]^{2} \mathrm{~d} x \\
& -2 \int_{-\infty}^{\infty} f(x) f_{N}(x) \mathrm{d} x .
\end{aligned}
$$

From the orthonormality relation,

$$
\int_{-\infty}^{\infty}\left[f_{N}(x)\right]^{2} \mathrm{~d} x=\sum_{i=0}^{N}\left(C_{i}\right)^{2}
$$

is obtained. The third term of the right-hand side of Eq. (7) is expressed by

$$
\begin{aligned}
\int_{-\infty}^{\infty} f(x) f_{N}(x) \mathrm{d} x= & \sum_{i=0}^{N} \int_{-\infty}^{\infty} f(x) C_{i} N_{i} \\
& \times H_{i}\left(\sqrt{2} \frac{x}{w}\right) \exp \left[-\left(\frac{x}{w}\right)^{2}\right] \mathrm{d} x \\
= & \sum_{i=0}^{N}\left(C_{i}\right)^{2}
\end{aligned}
$$

Consequently, with Eqs. (1), (8), and (9), Eq. (7) is reduced to

$$
I_{\text {Field }}(w)=1-\sum_{i=0}^{N}\left(C_{i}\right)^{2} .
$$

Because Eq. (5) leads to

$$
1=\sum_{i=0}^{\infty}\left(C_{i}\right)^{2} \geq \sum_{i=0}^{N}\left(C_{i}\right)^{2} \geq 0,
$$

$\sum_{i=0}^{N}\left(C_{i}\right)^{2}$ must be maximized to minimize $I_{\text {Field }}(w)$.

The Gaussian profile is the zeroth-order term of the Hermite-Gaussian function. Therefore the zerothorder term of Eq. (2) is necessary for approximating the field profile by a least-squares fit to the Gaussian profile. To minimize $I_{\text {Field }}(w), C_{0}{ }^{2}$ must be maximized, that is, $C_{0}$ must approach 1 . When $C_{0}$ is maximized, the field profile of the fundamental mode in a waveguide is best fitted to the Gaussian profile from the viewpoint of a least-squares fit. Accordingly, we can define the spot size by $w$, giving the 
maximum value of $C_{0}$. Because $C_{0}$ is given by

$$
C_{0}=\frac{1}{w^{1 / 2}\left(\frac{\pi}{2}\right)^{1 / 4}} \int_{-\infty}^{\infty} \exp \left[-\left(\frac{x}{w}\right)^{2}\right] f(x) \mathrm{d} x
$$

the condition to maximize Eq. (12), i.e.,

$$
\frac{\partial}{\partial w} C_{0}=0
$$

gives the following formula:

$$
w=\left\{\frac{4 \int_{-\infty}^{\infty} x^{2} \exp \left[-\left(\frac{x}{w}\right)^{2}\right] f(x) \mathrm{d} x}{\int_{-\infty}^{\infty} \exp \left[-\left(\frac{x}{w}\right)^{2}\right] f(x) \mathrm{d} x}\right\}^{1 / 2} .
$$

Equation (14) is the most fundamental form of the new spot-size formula that we propose in this work.

Because Eq. (14) includes $w$ on the right-hand side, we need an explicit or recursive expression to calculate $w$ numerically. Because the evaluation of the spot size is equivalent to the approximation of $f(x)$ by a Gaussian function, the Gaussian function on the right-hand side can be approximated by

$$
\exp \left[-\left(\frac{x}{w}\right)^{2}\right] \simeq f(x)
$$

With relation (15), Eq. (14) can be approximated by

$$
w^{(0)} \simeq\left\{\frac{4 \int_{-\infty}^{\infty} x^{2}[f(x)]^{2} \mathrm{~d} x}{\int_{-\infty}^{\infty}[f(x)]^{2} \mathrm{~d} x}\right\}^{1 / 2} .
$$

Relation (16) is the same as the formula based on the quadratic moment of optical power profile. ${ }^{10}$ However, if we use the successive approximation for Eq. (14), we can calculate a more accurate value of the spot size. First we calculate the spot size $w^{(0)}$ by relation (16) and substitute $w^{(0)}$ into $w$ on the righthand size of Eq. (14). The obtained spot size is again substituted into $w$ on the right-hand side to get the successive approximation. The repetition of this process gives the following recursive formula:

$$
w^{(v+1)}=\left|\frac{4 \int_{-\infty}^{\infty} x^{2} \exp \left\{-\left[\frac{x}{w^{(v)}}\right]^{2}\right\} f(x) \mathrm{d} x}{\int_{-\infty}^{\infty} \exp \left\{-\left[\frac{x}{w^{(v)}}\right]^{2}\right\} f(x) \mathrm{d} x}\right|^{1 / 2},
$$

where $v$ is the order of successive approximation.
Then, starting from $w^{(0)}$ as calculated by relation (16) and using Eq. (17), we can get a more accurate spot size. This successive approximation is one of the merits of our formula.

\section{B. Formulas in Near-Field and Far-Field Patterns}

In general, the optical power distribution of the fundamental mode of a waveguide is measured more easily from a far-field pattern (FFP) than from a near-field pattern (NFP). Therefore a formula for calculating the spot size from a FFP is necessary as well as that for a NFP. Assuming the coordinate system shown in Fig. 1, the Fourier transform of Eq. (14) that corresponds to the Fraunhofer transform yields

$$
W=\left\{\frac{4 \int_{-\infty}^{\infty} \Omega^{2} \exp \left[-\left(\frac{\Omega}{W}\right)^{2}\right] F(\Omega) \mathrm{d} \Omega}{\int_{-\infty}^{\infty} \exp \left[-\left(\frac{\Omega}{W}\right)^{2}\right] F(\Omega) \mathrm{d} \Omega}\right\}^{1 / 2},
$$

where $F(\Omega)$ is the Fourier transform of $f(x), \Omega=k_{0} x^{\prime} / z$, $k_{0}=2 \pi / \lambda, x^{\prime}$ is the transverse coordinate in a FFP, $z$ is the distance from the output end of waveguide to a FFP, $W$ is the spot size in a FFP, and $W=2 / w$.

When Eq. (18) is compared with Eq. (14), it is easily seen that our formula keeps its form against the Fourier transform. Table 1 summarizes our formula in a NFP and a FFP, comparing them with the formula of Villuendas et al. ${ }^{8}$ and the formula based on the quadratic moment. ${ }^{10,11}$ This table shows that the forms of two conventional formulas in the NFP are different from those in the FFP. Therefore it can be concluded that our formula is mathematically self-consistent. On the other hand, it is interesting to note that the formula based on the quadratic moment in the FFP has the same form as the formula of Villuendas et al. ${ }^{8}$ in the NFP and vice versa.

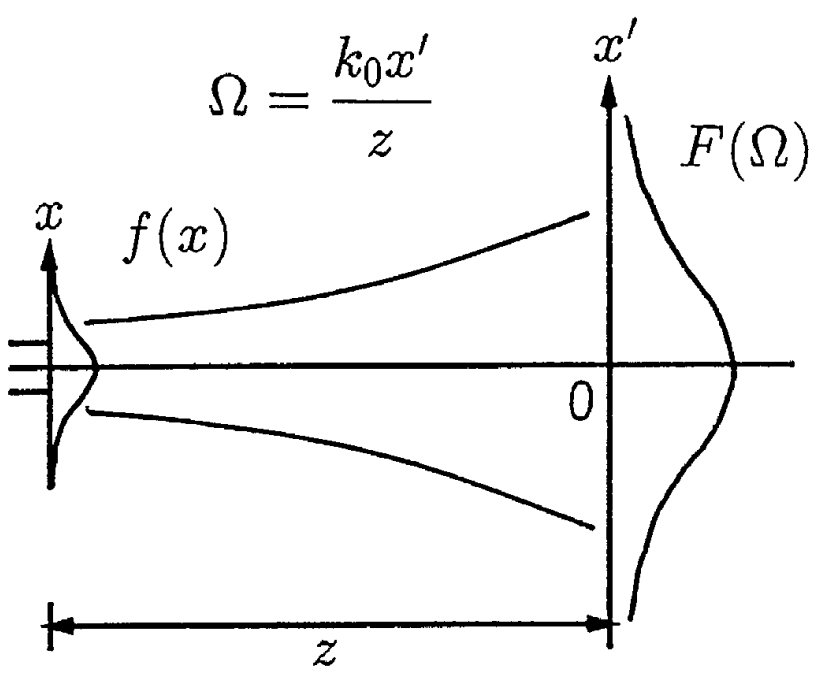

Fig. 1. Relation between NFP and FFP coordinates. 


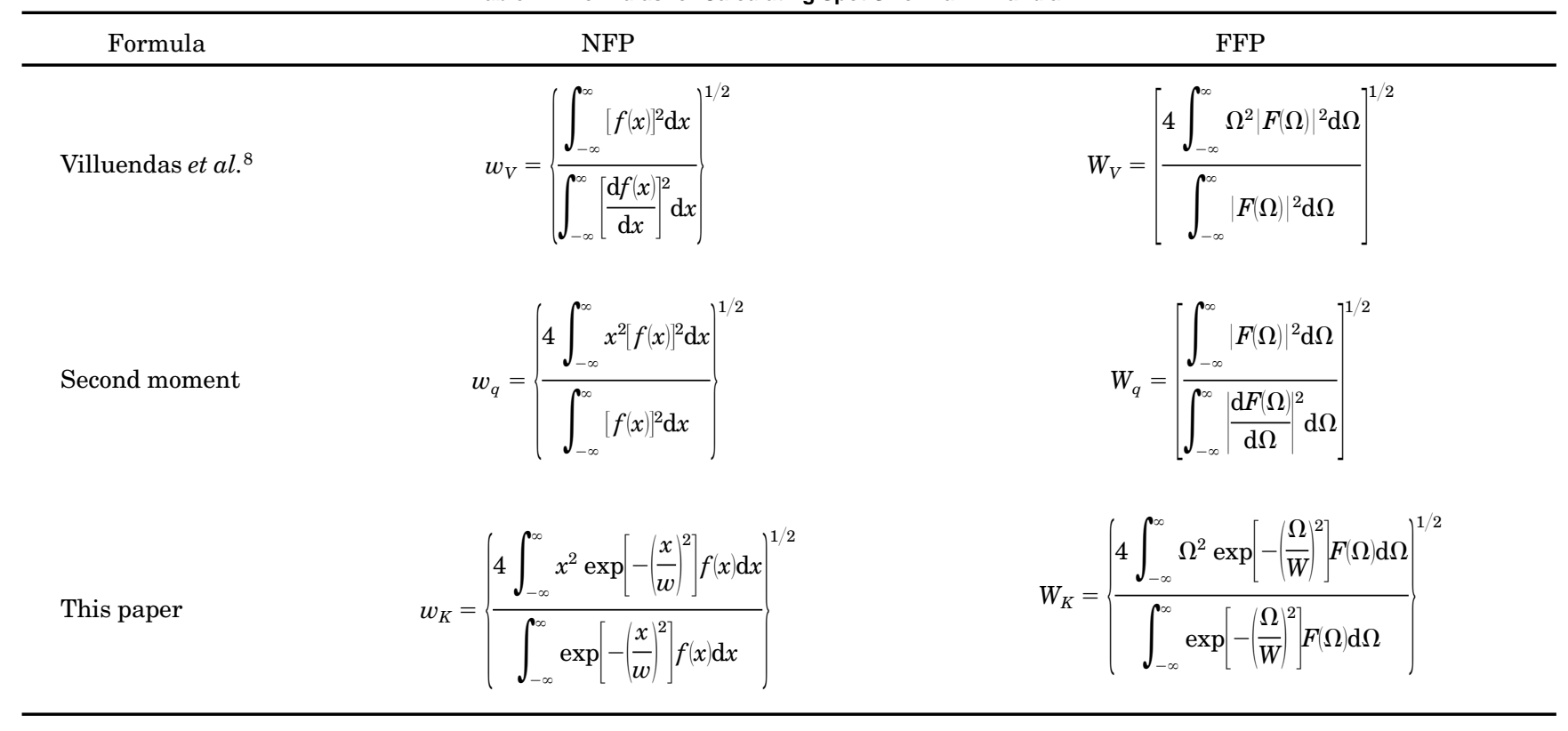

Because Eq. (18) also involves $W$ on the right-hand side, the Gaussian function is approximated by

$$
\exp \left[-\left(\frac{\Omega}{W}\right)^{2}\right] \simeq F(\Omega)
$$

Substituting relation (19) into Eq. (18), we obtain the following formula as the zeroth-order approximation:

$$
W=\left\{\frac{4 \int_{-\infty}^{\infty} \Omega^{2}[F(\Omega)]^{2} \mathrm{~d} \Omega}{\int_{-\infty}^{\infty}[F(\Omega)]^{2} \mathrm{~d} \Omega}\right\}^{1 / 2} .
$$

Here $[F(\Omega)]^{2}$ corresponds to the power distribution in the FFP. The successive approximation can also be executed with Eqs. (18) and (20) in the NFP.

\section{New Formula for Calculating Splice Loss between Two Waveguides}

We now consider the case in which two different waveguides that have different spot sizes are butt coupled with offset $\delta$, as shown in Fig. 2. $f_{1}(x)$ is the transverse field profile of waveguide 1 and $f_{2}(x-\delta)$ is that of waveguide 2. Both are assumed to be normalized from Eq. (1). The coupling efficiency is expressed by

$$
\eta(\delta)=\left[\int_{-\infty}^{\infty} f_{1}(x) f_{2}(x-\delta) \mathrm{d} x\right]^{2} .
$$

Substituting the field profiles expanded up to the second order of Eq. (2) into Eq. (21), after some cumbersome calculations, we get the following equa- tion:

$$
\begin{aligned}
& \eta(\delta)=\exp \left(-\frac{2 \delta^{2}}{A}\right)\left(\frac{2 B}{A}\right)\left\{C_{0}{ }^{(1)} C_{0}{ }^{(2)}+\left(\frac{6 B^{2}}{A^{2}}-\frac{1}{2}\right) C_{2}{ }^{(1)} C_{2}{ }^{(2)}\right. \\
& +\frac{1}{\sqrt{2}}\left[\frac{w^{(1)^{2}}-w^{(2)^{2}}}{A}\right]\left[C_{0}{ }^{(1)} C_{2}^{(2)}-C_{2}{ }^{(1)} C_{0}{ }^{(2)}\right] \\
& +\frac{2 \sqrt{2}}{A^{2}}\left[\frac{1}{\sqrt{2}}\left(A-\frac{12 B^{2}}{A}\right) C_{2}{ }^{(1)} C_{2}^{(2)}+w^{(2)^{2}} C_{0}^{(1)} C_{2}^{(2)}\right. \\
& \left.\left.+w^{(1)^{2}} C_{2}^{(1)} C_{0}{ }^{(2)}\right] \delta^{2}+\frac{8 B^{2}}{A^{4}} C_{2}{ }^{(1)} C_{2}{ }^{(2)} \delta^{4}\right\}^{2}, \\
& f_{1}(x)
\end{aligned}
$$

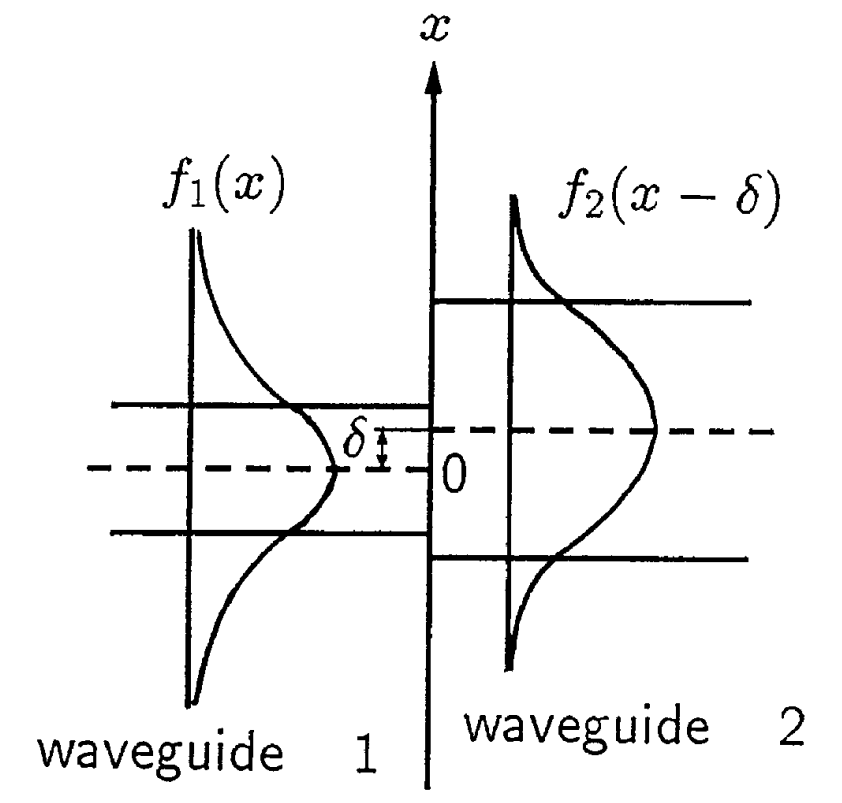

Fig. 2. Butt-coupling of waveguide 1 and waveguide 2 with offset $\delta\left[f_{1}(x)\right.$ and $f_{2}(x-\delta)$ are field profiles $]$. 
where $A=w^{(1)^{2}}+w^{(2)^{2}}, B=w^{(1)} w^{(2)}$, and the superscripts (1) and (2) indicate waveguides 1 and 2 of Fig. 2, respectively. Equation (22) implies that the splice loss between different waveguides with offset should be evaluated not only by the use of the spot size, as in the conventional case, but also by the use of the expansion coefficient.

If waveguide 1 and waveguide 2 have the same field profile, i.e., $f_{1}(x)=f_{2}(x)$, Eq. $(22)$ can be reduced to

$$
\begin{aligned}
\eta(\delta)= & \exp \left[-\left(\frac{\delta}{w}\right)^{2}\right] \\
& \times\left[\left[\left(C_{0}\right)^{2}+\left(C_{2}\right)^{2}\right]^{2}+2\left[\left(C_{0}\right)^{2}+\left(C_{0}\right)^{2}\right]\right. \\
& \times\left[\sqrt{2} C_{0} C_{2}-2\left(C_{2}\right)^{2} \mid \frac{\delta}{w}\right)^{2} \\
& +\left[\left(C_{0}\right)^{2}+\left(C_{2}\right)^{2}\right]\left(C_{2}\right)^{2} \\
& \left.+\left[\sqrt{2} C_{0} C_{2}-2\left(C_{2}\right)^{2}\right]^{2}\right]\left(\frac{\delta}{w}\right)^{4} \\
& \left.+\left[\sqrt{2} C_{0} C_{2}-2\left(C_{2}\right)^{2}\right]\left(C_{2}\right)^{2}\left(\frac{\delta}{w}\right)^{6}+\frac{\left(C_{2}\right)^{4}}{4}\left(\frac{\delta}{w}\right)^{8}\right) .
\end{aligned}
$$

Now we fit Eq. (23) to the splice loss of a Gaussian profile with offset $\delta$, as given by

$$
\eta_{G}(\delta)=\exp \left[-\left(\frac{\delta}{w}\right)^{2}\right]
$$

In the same way we used to derive Eq. (14), we consider the squared error $I_{\text {splice }}(w)$ between Eqs. (23) and $(24)$ as

$$
I_{\text {splice }}(w)=\int_{-\infty}^{\infty}\left[\eta(\delta)-\eta_{G}(\delta)\right]^{2} \mathrm{~d} \delta
$$

In Appendix A, we describe the equivalence of the maximization of $C_{0}$ and $C_{2}=0$. Therefore when the spot size $w$ satisfies Eq. (14), all terms involving $C_{2}$ in Eq. (23) can be eliminated, and Eq. (23) is reduced to

$$
\eta(\delta)=\exp \left[-\left(\frac{\delta}{w}\right)^{2}\right]\left(C_{0}\right)^{4}
$$

From Eqs. (24)-(26), the equivalence of the minimization of $I_{\text {splice }}(w)$ and the maximization of $C_{0}$ can be easily derived. Accordingly the least-squares fit of the mode profile $f(x)$ to the Gaussian beam is equivalent to that of the splice loss $\eta(\delta)$ to the splice loss of Gaussian beam. Our formula, Eq. (14), gives the least-squares approximation of both the field profile and the splice loss to those of the Gaussian profile.

\section{Accuracy of Spot-Size Definitions}

A. Effectiveness of Successive Approximation

In this section we discuss the effectiveness of the successive approximation described in Subsection 2.A. In this paper, $v$ is used as the number of successive approximations.

Figure 3 shows the error of spot size normalized by the half-thickness of core $a$ versus the number of successive approximations $\nu$. The value labeled EXACT in the figures means the spot size given by the definition of the maximizing of the zeroth-order expansion coefficient $C_{0}$. We calculate the exact spot size $w_{\text {exact}}$, which maximizes Eq. (12), by the numerical integration and the iterative division method. The accuracy of numerical integration is better than $10^{-14}$ and the accuracy of the iteration division method is better than $10^{-9}$. In this evaluation, slab waveguides for which $V$ values are $0.7,1.2$, and 1000 are assumed. From Fig. 3, it is seen that the error of normalized spot size decreases exponentially against the number of successive approximations $v$. Accordingly, we can obtain an accurate spot size by the successive-approximation method.

Let us define the error $E^{(v)}$ after $v$ th successive approximations by

$$
E^{(v)} \equiv w^{(v)}-w_{\text {exact }}
$$

It can be seen from Fig. 3 that the error $E^{(v)}$ decreases exponentially with increasing $v$ because $\mid \log E^{(v) \mid}$ decreases linearly. This means that the exact value of $w$ can be predicted from $w^{(v)}$ for three different numbers of $\nu$. We designate these three numbers as $n-2, n-1$, and $n$. This predicted value of $w_{\text {exact }}$ is calculated from the following equation:

$$
w_{\text {exact }}=\frac{1}{e^{k}-1}\left[w^{(n-2)} e^{k}-w^{(n-1)}\right]
$$

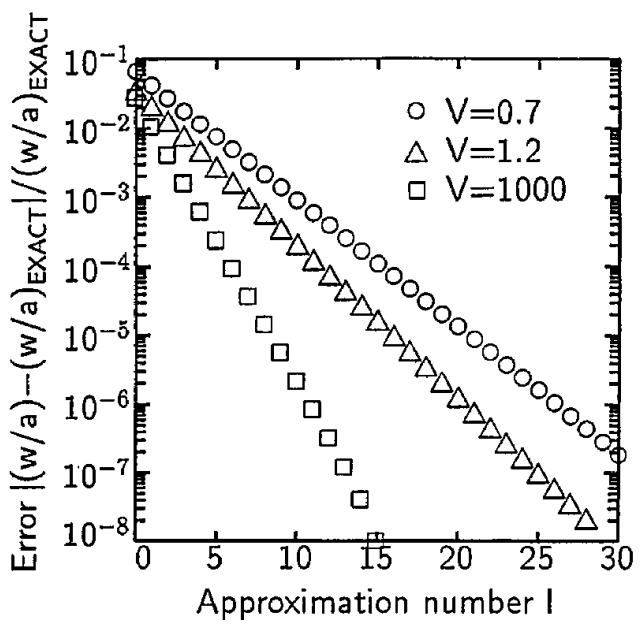

Fig. 3. Error in normalized spot size versus number of successive approximations $(V=0.7,1.2,1000)$. 
where the constant $k$ is given by

$$
k=\ln \frac{w^{(n)}-w^{(n-1)}}{w^{(n-1)}-w^{(n-2)}} .
$$

Because the values of $w^{(n-2)}$ and $w^{(n-1)}$ are already calculated before the calculation of $w^{(n)}$, this evaluation of the exact value of $w$ can be performed with only the values of $w^{(v)}$ calculated before the $n$ th-order successive approximation. In other words, a more accurate spot size can be obtained with the values of former orders of successive approximation than with only the value of successive approximation of order $n$.

Now let us examine the accuracy of Eq. (28). In this section we consider the following cases:

Case A, no successive approximation: $\quad v=0$;

Case $B$, second-order successive approximation: $v=2$;

Case $\mathrm{C}$, error prediction in which orders $0-2$ are used:

$$
k=\ln \frac{w^{(2)}-w^{(1)}}{w^{(1)}-w^{(0)}}, \quad w_{\text {exact }}=\frac{1}{e^{k}-1}\left[w^{(0)} e^{k}-w^{(1)}\right]
$$

Case D, error prediction in which orders $3-5$ are used:

$$
k=\ln \frac{w^{(5)}-w^{(4)}}{w^{(4)}-w^{(3)}}, \quad w_{\text {exact }}=\frac{1}{e^{k}-1}\left[w^{(3)} e^{k}-w^{(4)}\right) .
$$

Figure 4 shows the error of calculated spot size versus $V$ value. From this figure it is obvious that, even if the number of successive approximations is the same for case B and case C, case C, which uses Eqs. (28) and

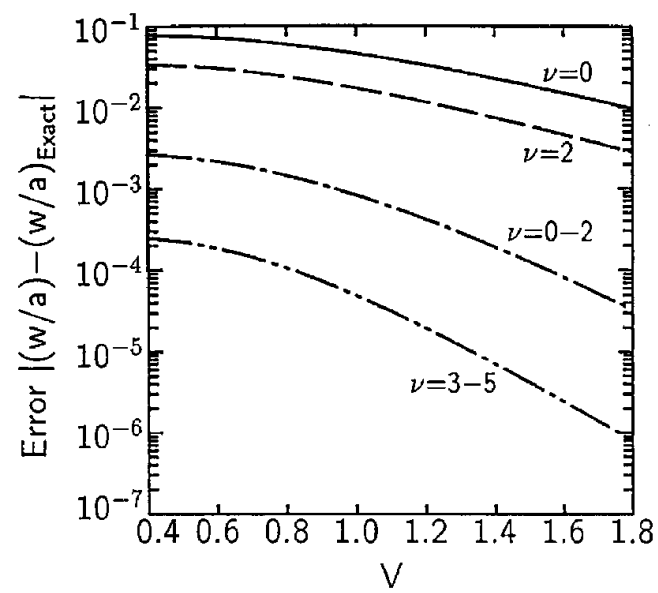

Fig. 4. Accuracy of normalized spot size calculated by various numbers of successive approximations.
(29), is more accurate by several orders than case B. When Eqs. (28) and (29) are used, the accuracy is better than $\sim 10^{-3}$ by the second-order successive approximation in almost whole range of $V$ values and is better than $\sim 10^{-4}$ by the fifth-order successive approximation.

\section{B. Comparison of Various Formulas}

In this section we compare the spot sizes that are calculated from various formulas with the exact value. Compared formulas are described as follows:

(1) The $1 / e$ half-width of the field distribution $\left(w_{e}\right)$ :

$$
f\left(w_{e}\right)=f(0) / e ;
$$

(2) Formula of Villuendas et $a l .{ }^{8}\left(w_{V}\right)$ :

$$
w_{V}=\left\{\frac{\int_{-\infty}^{\infty}[f(x)]^{2} \mathrm{~d} x}{\int_{-\infty}^{\infty}\left[\frac{\mathrm{d} f(x)}{\mathrm{d} x}\right]^{2} \mathrm{~d} x}\right\}^{1 / 2} ;
$$

(3) The formula based on the quadratic moment of power distribution ${ }^{10}\left(w_{q}\right)$ :

$$
w_{q}=\left\{\frac{4 \int_{-\infty}^{\infty} x^{2}[f(x)]^{2} \mathrm{~d} x}{\int_{-\infty}^{\infty}[f(x)]^{2} \mathrm{~d} x}\right\}^{1 / 2} ;
$$

(4) The formula of this paper (without successive approximation, $v=0)\left[w_{K}{ }^{(0)}\right]$ :

$$
w_{K}^{(0)}=\left\{\frac{4 \int_{-\infty}^{\infty} x^{2}[f(x)]^{2} \mathrm{~d} x}{\int_{-\infty}^{\infty}[f(x)]^{2} \mathrm{~d} x}\right\}^{1 / 2} .
$$

However, because the form of the third case is the same as that of our formula without successive approximation, the curves of both formulas in the figures below are identical.

(5) The formula of this paper after the first successive approximation $(v=1)\left[w_{K}^{(1)}\right]$ :

$$
w_{K}^{(1)}=\left|\frac{4 \int_{-\infty}^{\infty} x^{2} \exp \left\{-\left[\frac{x}{w_{K}^{(0)}}\right]^{2}\right\} f(x) \mathrm{d} x}{\int_{-\infty}^{\infty} \exp \left\{-\left[\frac{x}{w_{K}{ }^{(0)}}\right]^{2}\right\} f(x) \mathrm{d} x}\right|^{1 / 2} .
$$


Subsituting the field profile of a three-layer slab waveguide into Eqs. (32)-(35) and expressing the spot size as a function of normalized frequency $V$ and the normalized propagation constant $b$, we obtain the following formulas:
The formula of this paper with $v=0$ is more accurate than the others through almost the entire region of $V$ and has almost the same accuracy as that of the formula of Villuendas et al., ${ }^{8}$ even if $V$ is close to $\pi / 2$. However, our formula with $v=0$ has a several percent error.

$$
\begin{aligned}
\frac{w_{e}}{a} & = \begin{cases}1+\frac{1}{V \sqrt{b}}\left\{1+\ln \left[\cos V(1-b)^{1 / 2}\right]\right\} & \left(w_{e}>a\right) \\
\frac{1}{V(1-b)^{1 / 2}} \cos ^{-1} \frac{1}{e} & \left(w_{e} \leq a\right) .\end{cases} \\
\frac{w_{V}}{a} & =\left[\frac{\left.V+\frac{1}{\sqrt{b}}\right]^{1 / 2}}{V^{3}(1-b)},\right. \\
\frac{w_{K}{ }^{(0)}}{a} & =\frac{w_{q}}{a} \\
& =2\left[\frac{2 b \sqrt{b}(1-b) V^{3}+6 b(1-b) V^{2}+3 \sqrt{b}(2-3 b) V+3(1-2 b)}{6 V^{2}(1-b) b(V \sqrt{b}+1)}\right]^{1 / 2},
\end{aligned}
$$

where $V=k_{0} a n_{1} \sqrt{2 \Delta}, a$ is the half-thickness of the core, $n_{1}$ is the index of the core, $n_{2}$ is the cladding index, $\Delta=\left(n_{1}^{2}-n_{2}^{2}\right) / 2 n_{1}^{2}$ is the relative index difference of the core and the cladding, $b=$ $\left[\left(\beta / k_{0}\right)^{2}-n_{2}^{2}\right] /\left(n_{1}^{2}-n_{2}^{2}\right)$, and $\beta$ is the propagation constant and is evaluated from $V$ by the dispersion relation. The spot size is normalized by the halfthickness of core $a$.

Figure 5 compares the spot sizes that are evaluated from Eqs. (37)-(39) with the exact values.

Figure 5 implies that the $1 / e$ half-width of the field distribution has the worst accuracy among the formulas, and so this formula is not suitable for the precise evaluation of the spot size. The value of $C_{0}$ is close to the maximum value near $V=2.293$, and the error of the $1 / e$ definition becomes minimum. Because the field profile is best fitted to the Gaussian profile when $C_{0}$ is close to 1 , the $1 / e$ half-width approaches the spot size of a Gaussian beam. In this case, $V=2.293, C_{0}$ $=0.9995$, and $w / a=1.144$.

Although the formula of Villuendas et al. ${ }^{8}$ is the most accurate of the four formulas near $V=\pi / 2$ (except for our formula with $v=1$ ), their formula has a lower accuracy in the other region. In other words, their formula can evaluate the spot size with an error of $2 \%-3 \%$ for ordinary single-mode waveguides but has a large error for reduced-confinement waveguides $^{1}$ that have a large spot size with a small core thickness and for tight-confinement waveguides, including antiresonant reflecting optical waveguides ${ }^{13}$ whose field profile is similar to that of three-layer slab waveguide that has a very large $V$ value, e.g., $V=$ 1000 .
Figure 5 also shows the curve of our formula with $v=1$. It is seen that the accuracy is improved by the first-order successive approximation and becomes higher than that of any other formulas. Then, by a few successive approximations, the curve of our formula cannot be distinguished from that of the exact value in the linear scale.

From Fig. 5 we can derive a $V$ value that gives the minimum spot size of a waveguide that has a certain fixed refractive-index difference (see appendix B). This value is $V=1.185$, and the corresponding spot size normalized by the half-thickness of the core is $w / a=1.856$. Accordingly, if the materials of the waveguide are determined for a three-layer slab waveguide, the half-thickness of the core that minimizes the spot size is given automatically for a certain wavelength.

\section{Field Amplitude at the Point of the Spot Size}

In this section we consider the ratio of field amplitude at the point of the spot size to the maximum amplitude. We calculate the spot size against the $V$ value and the ratio of the field amplitude at the point of $w$ to the maximum amplitude $f(0)$. Figure 6 shows the result. The curve of our formula with $v=3-5$ is almost equal to that of the exact value. It can be seen that the field ratio is different from $1 / e$. The larger the $V$ value is, the larger $f(w) / f(0)$ is, and $f(w) / f(0)$ approaches 0.4494 . This fact means that, even if two waveguides have the same spot size, the field profiles are different if these waveguides have different $V$ values. 


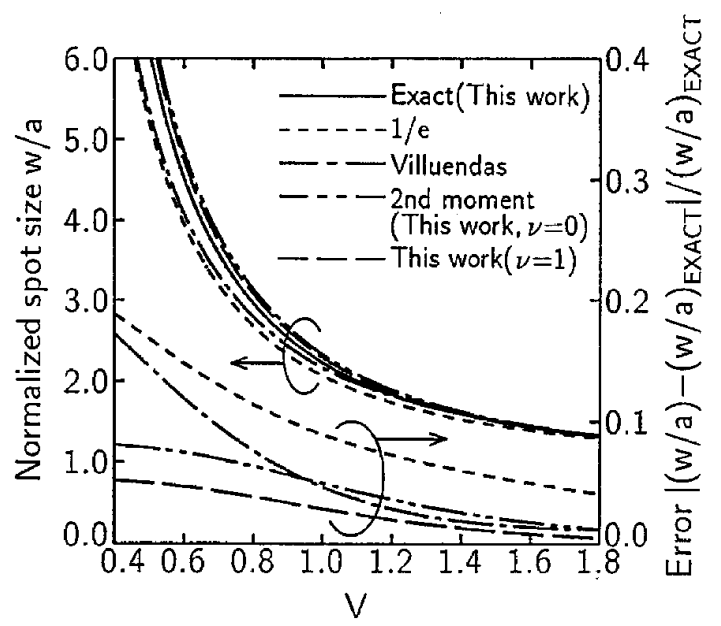

(a)

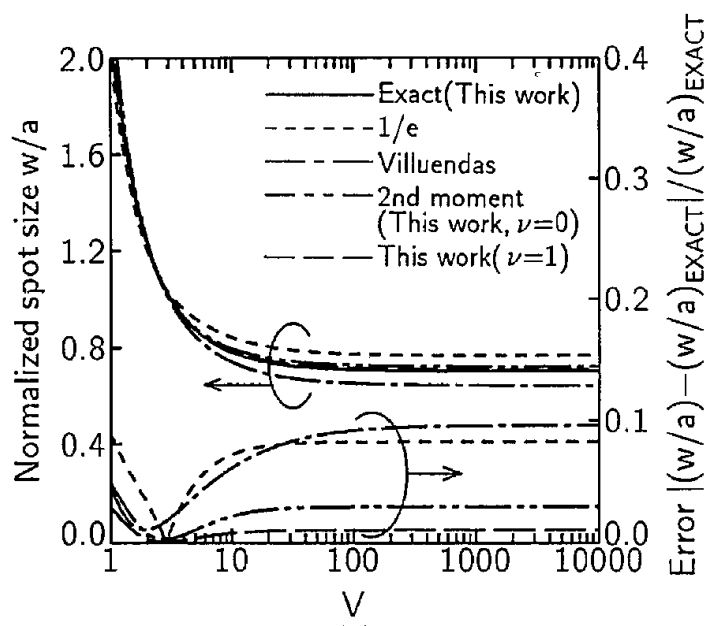

(b)

Fig. 5. Comparison of various spot-size formulas: (a) small $V$ value, (b) large $V$ value.

\section{Accuracy of Splice-Loss Formula}

\section{A. Introduction of Second-Order Term}

Here we again consider the case in which two different waveguides that have different spot sizes are butt coupled with offset $\delta$, as shown in Fig. 2. A formula that evaluates the coupling loss by the use of only the spot size has been used. This formula is derived from the overlap integral of two Gaussian beams that have different spot sizes and is given by

$$
\alpha_{\text {spot }}(\delta)=-10 \log \left[\left(\frac{2 B}{A}\right)^{2} \exp \left(-\frac{2 \delta^{2}}{A}\right)\right](\mathrm{dB})
$$

where $A$ and $B$ are the same as used in Eq. (22). If two waveguides that have the same spot sizes are butt coupled without offset $\delta$, i.e., $\delta=0$, the coupling loss is evaluated from Eq. (40) to be 0. As shown in Section 3, however, even if the spot sizes of two waveguides are the same, the field profiles are not necessarily equal. Therefore the coupling loss is not

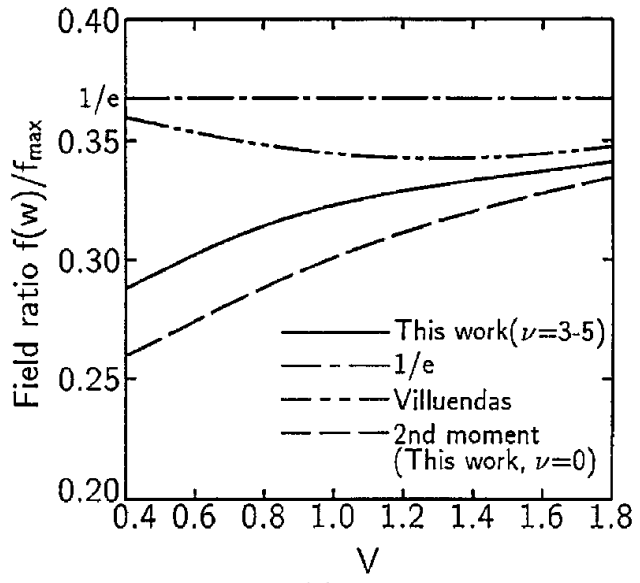

(a)

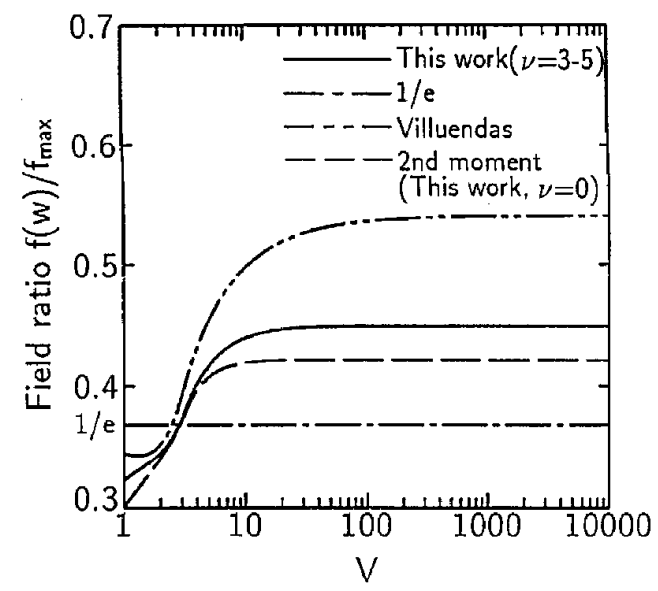

(b)

Fig. 6. Normalized field amplitude at the point of the spot size: (a) small $V$ value, (b) large $V$ value.

equal to 0, even if the offset $\delta=0$. In such cases, Eq. (40) leads to a large error.

We have proposed an analytical expression of the splice loss with offset, Eq. (22). The splice loss can be evaluated from the following equation:

$$
\alpha(\delta)=-10 \log [\eta(\delta)](\mathrm{dB})
$$

Now let us compare Eq. (41) with Eq. (40). Here we again investigate the TE fundamental mode of a symmetric three-layer slab waveguide. The field profile of a symmetric three-layer slab waveguide can be written in terms of $V$ and $b$ by

$$
f(x)= \begin{cases}\cos \left[\frac{V}{a}(1-b)^{1 / 2} x\right] & |x| \leq a \\ (1-b)^{1 / 2} \exp \left[-\frac{V}{a}(\sqrt{b}|x|-a)\right] & |x|>a .\end{cases}
$$




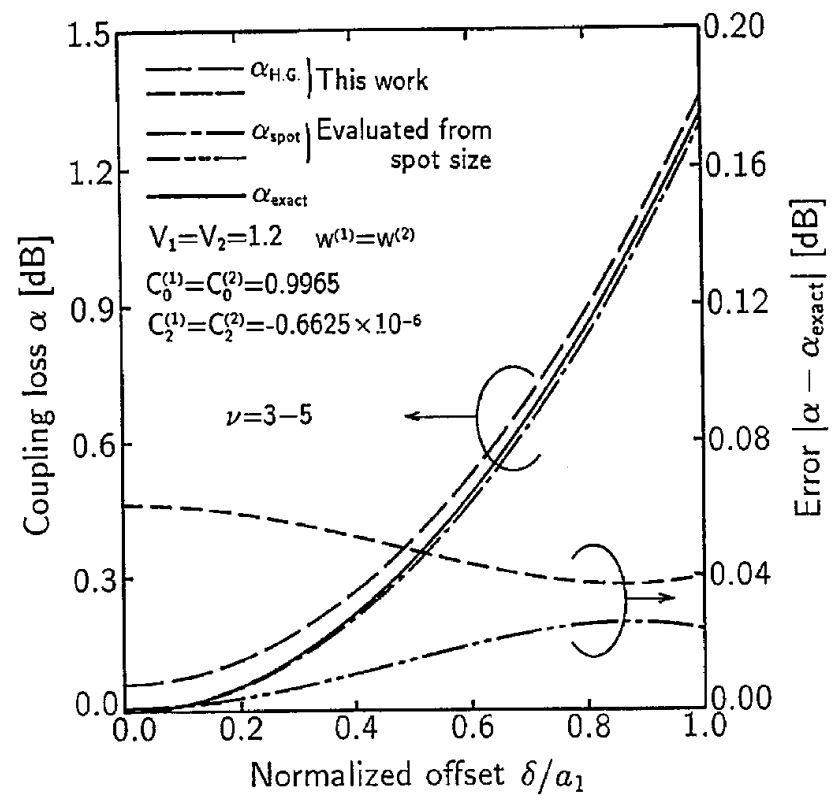

Fig. 7. Coupling loss of two waveguides that have the same spot size, evaluated without $C_{4}(V=1.2)$.

The exact value of offset splice loss is obtained by the numerical calculation of the following overlap integral:

$$
\alpha_{\text {exact }}(\delta)=-10 \log \left[\int_{-\infty}^{\infty} f_{1}(x) f_{2}(x-\delta) \mathrm{d} x\right]^{2}(\mathrm{~dB})
$$

Because our formula for calculating the spot size is the most accurate, we use our formula to evaluate Eq. (41). Because it is ensured that $C_{2} \simeq 0$ when our formula is used, all terms that involve $C_{2}$ are omitted in Eq. (41). Figure 7 includes the curves calculated from Eqs. (40), (41), and (43). This figure shows the coupling loss (left axis) and the error from the exact value (right axis) versus the normalized offset. The $V$ value of both waveguides is $V=1.2$. It can be seen that the error of our formula, Eq. (41), is larger than that of the conventional formula, Eq. (40). When the offset is equal to 0 , the exact coupling loss must be 0 . But our formula is not equal to 0 and has a large error of $0.1 \mathrm{~dB}$. This reason can be thought through as follows: If two waveguides are the same, our formula is Eq. (23). When the offset $\delta=0$, the coupling efficiency is given by $\left[\left(C_{0}\right)^{2}+\left(C_{2}\right)^{2}\right]^{2}$ according to Eq. (23). Because $C_{2}=0$ if our spot-size formula is used, $\left(\boldsymbol{C}_{0}\right)^{4}$ must be 1 to ensure that the coupling loss is 0 . However, such a condition is realized only when the field profile is identical to the Gaussian profile. This means that the field expansion in terms of Hermite-Gaussian functions up to the second order is not accurate enough, and higherorder terms are needed.
Table 2. Comparison of Various Expansion Coefficients

\begin{tabular}{cccc}
\hline$V$ & $C_{0}$ & $C_{2}$ & \multicolumn{1}{c}{$C_{4}$} \\
\hline 0.7 & 0.9907 & $-0.6490 \times 10^{-5}$ & 0.1260 \\
1.2 & 0.9965 & $-0.6625 \times 10^{-6}$ & 0.08133 \\
\hline
\end{tabular}

B. Introduction of the Fourth-Order Term

Therefore we introduce the fourth-order term $C_{4}$, which is given by

$$
\begin{aligned}
C_{4}= & \frac{1}{\sqrt{384} w^{1 / 2}\left(\frac{\pi}{2}\right)^{1 / 4}} \int_{-\infty}^{\infty}\left[64\left(\frac{x}{w}\right)^{4}-96\left(\frac{x}{w}\right)^{2}-12\right] \\
& \times \exp \left[-\left(\frac{x}{w}\right)^{2}\right] f(x) \mathrm{d} x .
\end{aligned}
$$

The values of some expansion coefficients for the cases of $V=0.7$ and 1.2 are given in Table 2 . Because $C_{2}$ is very small whereas $C_{4}$ is $\sim 10 \%$ of $C_{0}, C_{4}$ cannot be neglected. Here we consider the case in which the exact field profile of a three-layer slab waveguide is expanded in terms of Hermite-Gaussian functions. When $V=1.2$, the exact field profile of the TE fundamental mode is compared with the approximated field profiles in terms of only zerothorder Hermite-Gaussian functions and up to fourthorder Hermite-Gaussian functions. Figure 8 shows a comparison between the approximation for which only $C_{0}$ is used and that for which $C_{4}$ is used. It can be seen that the approximation in which only $C_{0}$ is used is not sufficiently accurate, and $C_{4}$ is necessary for improving the approximation. Accordingly, if the formula for calculating the splice loss includes $C_{4}$, it is expected that the accuracy of this equation is improved.

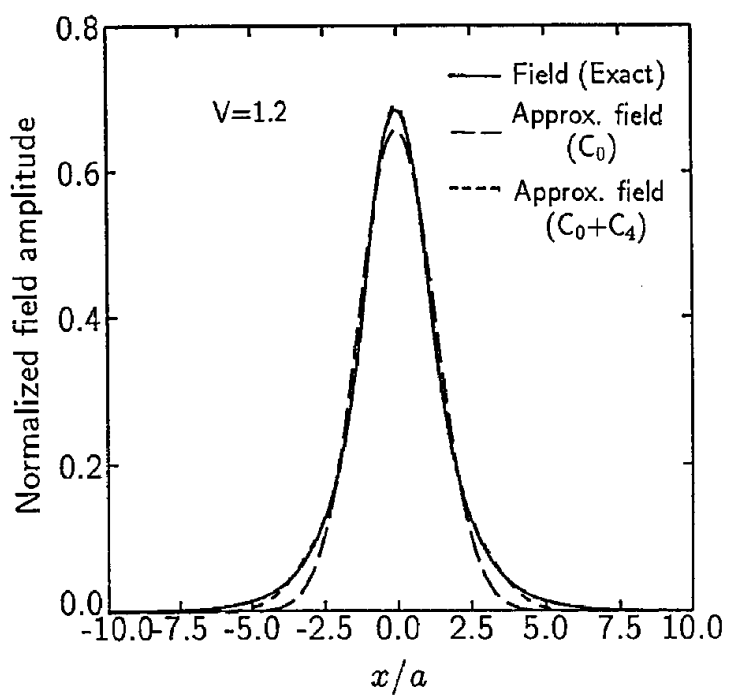

Fig. 8. Comparison of exact field profile and approximated Gaussian profile. 
Substituting the field profiles expanded up to fourth order in Eq. (2) into Eq. (21), after some cumbersome calculations, we obtain

$$
\begin{aligned}
& \eta_{\mathrm{HG}}(\delta)=\exp \left(-\frac{2 \delta^{2}}{A}\right)\left(\frac{2 B}{A}\right)\left(C_{0}{ }^{(1)} C_{0}{ }^{(2)}\right. \\
& +\frac{1}{8 A^{4}}\left(3 D-108 B^{2} E+338 B^{4}\right) C_{4}{ }^{(1)} C_{4}^{(2)} \\
& +\frac{3}{2 \sqrt{6} A^{2}} F\left[C_{0}^{(1)} C_{4}^{(2)}+C_{4}^{(1)} C_{0}^{(2)}\right] \\
& +\frac{2}{A^{3}}\left\{6 F\left[w^{(2) 2} C_{0}{ }^{(1)} C_{4}{ }^{(2)}-w^{(1) 2} C_{4}{ }^{(1)} C_{0}{ }^{(2)}\right]\right. \\
& -\frac{1}{24 A^{2}}\left(35 D-1296 B^{2} E\right. \\
& \left.\left.+4058 B^{4}\right) C_{4}^{(1)} C_{4}{ }^{(2)}\right\} \delta^{2} \\
& +\frac{8}{A^{4}}\left\{\frac{1}{4 A^{2}}\left(D-46 B^{2} E+186 B^{4}\right) C_{4}{ }^{(1)} C_{4}^{(2)}\right. \\
& \left.+\frac{1}{\sqrt{6}}\left[w^{(2)^{4}} C_{0}{ }^{(1)} C_{4}{ }^{(2)}+w^{(1)^{4}} C_{4}{ }^{(1)} C_{0}{ }^{(2)}\right]\right] \delta^{4} \\
& +\frac{16 B^{2}}{3 A^{7}}\left(3 E-22 B^{2}\right) C_{4}^{(1)} C_{4}^{(2)} \delta^{6} \\
& +\left.\frac{32 B^{4}}{3 A^{8}} C_{4}^{(1)} C_{4}^{(2)} \delta^{8}\right|^{2},
\end{aligned}
$$

where $w^{(1)^{2}}+w^{(2)^{2}}=A, w^{(1)} w^{(2)}=B, w^{(1)^{8}}+w^{(2)^{8}}=D, w^{(1)^{4}}$ $+w^{(2)^{4}}=E$, and $w^{(1)^{2}}-w^{(2)^{2}}=F$. Because the

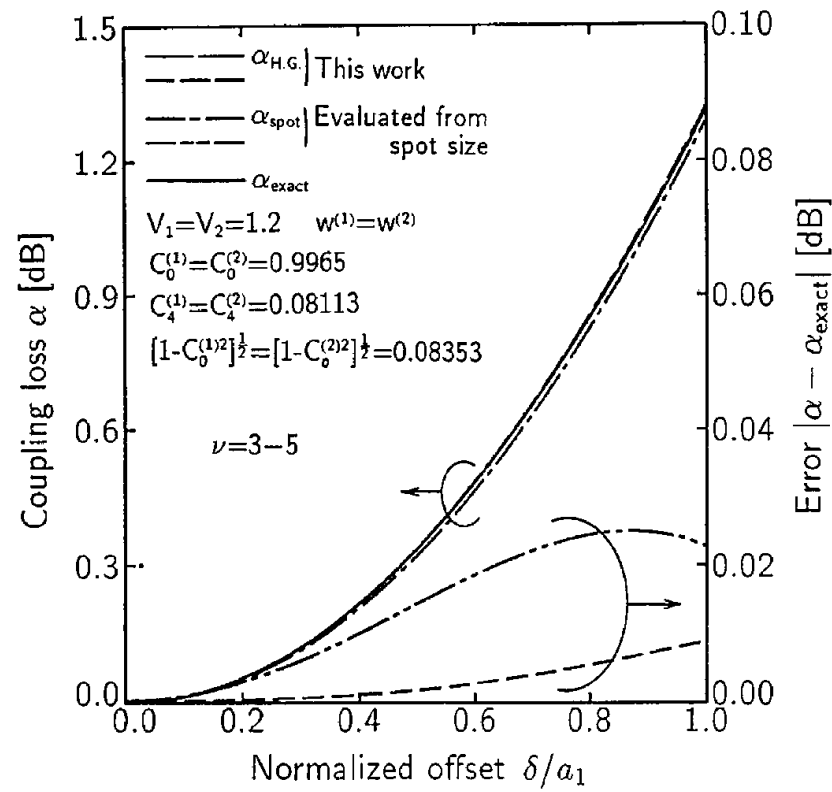

Fig. 9. Coupling loss of two waveguides that have the same spot size, evaluated with $C_{4}\left[w^{(1)}=w^{(2)}\right]$.

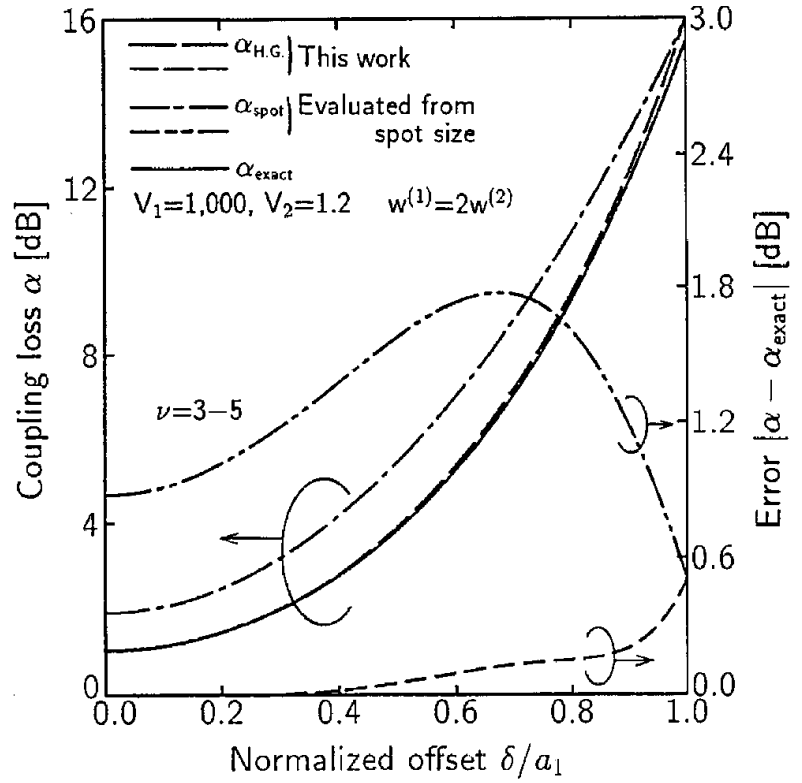

Fig. 10. Coupling loss of two waveguides that have different spot sizes, evaluated with $C_{4}\left[w^{(1)}=2 w^{(2)}\right]$.

number of required parameters should be as few as possible, the following approximation is used. Let us assume the inequality

$$
\left|C_{0}\right|>\left|C_{4}\right| \gg\left|C_{2}\right|,\left|C_{6}\right|,\left|C_{8}\right| \cdots .
$$

Thus, from Eq. (5), $C_{4}$ can be approximated by

$$
\left|C_{4}\right| \approx\left[1-\left(C_{0}\right)^{2}\right]^{1 / 2} .
$$

The sign of $C_{4}$ can be determined from the field profile (see Appendix C.) Figure 9 shows the coupling loss calculated from Eq. (45), in which the $V$ values of two waveguides are equal. Figure 10 shows the case of two different waveguides that have different spot sizes. Both figures show that our formula is more accurate than the conventional formula. Although $C_{4}$ is used in our formula, the coupling loss is evaluated by the use of only the spot size and $C_{0}$, because $C_{4}$ is calculated from $C_{0}$ by approximation (47).

To examine the accuracy of our formula, we calculated the coupling loss characteristics for many combinations of three-layer slab waveguides that have different $V$ values and spot sizes, and the result were compared with the exact values. From this consideration, it was confirmed that our formula is much more accurate than the conventional formula for almost all combinations of two waveguides. In particular, the error of the conventional formula reaches $0.2-1.0 \mathrm{~dB}$ for two different waveguides for which spot sizes are different by a factor of 2 , whereas the error of the formula of this paper is less than $0.1 \mathrm{~dB}$.

\section{Conclusion}

In this paper we defined the spot size by best fitting the field profile to the Gaussian profile from the viewpoint of a least-squares fit. Next, the field pro- 
file of fundamental mode in a waveguide was expanded in terms of Hermite-Gaussian functions, and a new precise recursive formula for calculating the spot size was derived. In addition, approximating the offset coupling loss of two waveguides by this expansion and using our spot-size formula, we best fitted the offset coupling loss to that of a Gaussian profile. Our spot-size formula can approximate more accurately by successive approximation and keeps its form against the Fourier transform. By using the characteristic that the error of normalized spot size decreases exponentially against the number of successive approximation, we can predict the precise spot size from a few orders of successive approximations.

Next we proposed a new formula for calculating splice loss between two waveguides and examined the accuracy of this formula. Although our formula includes zeroth- and fourth-order expansion coefficients in addition to spot size, the accuracy is extremely improved compared with that of the conventional formula. Because the fourth-order expansion coefficient can be calculated from that of the zeroth order, only two parameters, i.e., spot size and the zerothorder expansion coefficient are needed for the formula of this paper. When two different waveguides are butt coupled, the error of the coupling loss calculated by the conventional formula reaches as high as $0.2-$ $1.0 \mathrm{~dB}$. Even in this case, the formula of this paper can give much better accuracy.

In this paper we assumed the field profile to be symmetric with respect to the optical axis, and so only the even-order terms are involved in the expansion. As shown in Appendix A, the condition of maximizing $C_{0}$ is equivalent to making $C_{2}=0$. From this equivalence, it is concluded that when the field profile involves a second-order Hermite-Gaussian term, our formula will fail to give an accurate value of the spot size. This case corresponds to the field profile that has a local maximum elsewhere at $x=0$.

Although we assumed a symmetric field distribution in this paper, the case of asymmetric field distributions often occurs in integrated optical waveguides. In this case, we can treat the asymmetry by introducing the first-order expansion coefficient of HermiteGaussian expansion. The formula for the asymmetric case is being studied and will be presented in the future.

\section{Appendix A.}

The condition of making $C_{0}$ maximum is equivalent to $C_{2}=0$. This equivalence can be easily proved. Because $C_{2}$ is defined by

$$
\begin{aligned}
C_{2}= & \frac{1}{\sqrt{2} w^{1 / 2}\left(\frac{\pi}{2}\right)^{1 / 4}} \int_{-\infty}^{\infty}\left[4\left(\frac{x}{w}\right)^{2}-1\right] \\
& \times \exp \left[-\left(\frac{x}{w}\right)^{2}\right] f(x) \mathrm{d} x,
\end{aligned}
$$

we derive Eq. (14) directly by putting $C_{2}=0$. Thus the spot size calculated from Eq. (14) gives the maximum value of $C_{0}$ and $C_{2}=0$.

\section{Appendix B.}

Because it can be seen from Fig. 5 that $w / a$ is a function of can be $V$, we can put

$$
(w / a)=f(V) .
$$

Equation (B1) can be rewritten as

$$
w=a f(V) .
$$

By differentiating Eq. (B2) with respect to $V$ and putting the differential equal to 0 , we can obtain the condition to minimize the spot size from

$$
\frac{\partial a}{\partial V} f(V)+a \frac{\partial f(V)}{\partial V}=0 .
$$

With the definition of the $V$ value, Eq. (B3) becomes

$$
\frac{f(V)}{V}+\frac{\partial f(V)}{\partial V}=0
$$

We can obtain the $V$ value that minimizes the spot size by solving Eq. (B4).

Because Eq. (B4) is an implicit function of $V$, the $V$ value that minimizes the spot size is independent of the half-thickness of core $a$ and $\Delta$ for the symmetric three-layer slab waveguide. When Eq. (B4) is solved numerically, $V=1.185$ is obtained.

\section{Appendix C.}

The fourth-order term of Hermite-Gaussian expansion $g_{4}(x)$ is given by

$$
\begin{aligned}
g_{4}(x)= & \frac{C_{4}}{2 \sqrt{6} w^{1 / 2}\left(\frac{\pi}{2}\right)^{1 / 2}}\left[16\left(\frac{x}{w}\right)^{4}-24\left(\frac{x}{w}\right)^{2}+3\right] \\
& \times \exp \left[-\left(\frac{x}{w}\right)^{2}\right] .
\end{aligned}
$$

This equation has three maximums and two minimums when $C_{4}>0$. The values of $x$ that give maximums and minimums are

$$
x=0, \pm 0.760 w, \pm 1.71 w .
$$

Because the difference between the exact field profile $f(x)$ and the field $f_{0}(x)$ approximated by only the zeroth-order Hermite-Gaussian term is attributed to the fourth-order term, we use the value $J$, as defined by Eq. (C3), to judge the sign of $C_{4}$ :

$$
\begin{aligned}
J= & {\left[f(0)-f_{0}(0)\right]+2 \alpha_{1}\left[f(0.760 w)-f_{0}(0.760 w)\right] } \\
& +2 \alpha_{2}\left[f(1.71 w)-f_{0}(1.71 w)\right],
\end{aligned}
$$

where

$$
\alpha_{1}=\frac{g_{4}(0.760 w)}{g_{4}(0)}=-1.03
$$




$$
\alpha_{2}=\frac{g_{4}(1.71 w)}{g_{4}(0)}=1.25
$$

If the sign of $J$ is minus, the sign of $C_{4}$ is minus and vice versa.

\section{References}

1. K. Kasaya, O. Mitomi, M. Naganuma, Y. Kondo, and Y. Noguchi, "A simple laterally tapered waveguide for low-loss coupling to single-mode fibers," Photon. Tech. Lett. 5, 345-347 (1993).

2. K. Miura, I. Sawaki, and H. Nakajima, "Low-loss single-mode plastic waveguide fabricated by photopolymerization," in Integrated Guided-Wave Optics, Vol. 5 of 1988 OSA Technical Digest Series (Optical Society of America, Washington, D.C., 1988), pp. 58-61.

3. H. Yanagawa, T. Shimizu, S. Nakamura, and I. Ohyama, "Index- and 3-dimensional taper and its application to photonic devices," J. Lighwave Technol. 10, 587-592 (1992).

4. N. Yamaguchi, Y. Kokubun, and K. Sato, "Low-loss spot-size transformer by dual-taped waveguides (DTW-SST)," J. Lightwave Technol. 8, 587-594 (1990).

5. Y. Kokubun, S. Tamura, and T. Kondo, "Spot size transformer with a type-B antiresonant reflecting optical waveguide," Opt. Lett. 17, 1746-1748 (1992).

6. Y. Shani, C. H. Henry, R. C. Kistler, K. J. Orlowsky, and D. A.
Ackerman, "Efficient coupling of a semiconductor laser to an optical fiber by means of a tapered waveguide on silicon,"Appl. Phys. Lett. 55, 2389-2391 (1989).

7. N. Yoshimoto, K. Kawano, H. Takeuchi, S. Kondo, and Y. Noguchi, "Efficient coupling semiconductor waveguide spotsize converter with a double core structure," in Fourth Conference on Optoelectronics (Institute of Electronics, Information and Communication Engineers, Tokyo, 1992), pp. 10-11.

8. F. Villuendas, F. Calvo, and J. B. Marques, "Measurement of mode field radius in axially nonsymmetrical single-mode fibers with arbitrary power distribution," Opt. Lett. 12, 941943 (1987).

9. K. Petermann, "Constraints for fundamental-mode spot size for broadband dispersion-compensated single-mode fibers," Electron. Lett. 19, 712-714 (1983).

10. K. Hayata, M. Koshiba, and M. Suzuki, "Modal spot size of axially nonsymmetrical fibers," Electron. Lett. 22, 127-129 (1986).

11. P. A. Belanger, "Beam propagation and the $A B C D$ ray matrices," Opt. Lett. 16, 196-198 (1991).

12. D. Marcuse, "Loss analysis of single-mode fiber splices," Bell Syst. Tech. J. 56, 703-718 (1977).

13. T. Baba and Y. Kokubun, "Dispersion and radiation loss characteristics of antiresonant reflecting optical waveguidesnumerical results and analytical expressions," IEEE J. Quantum Electron. 28, 1689-1700 (1992). 